Pacific

Journal of

Mathematics

MULTIPLIERS OF OPERATOR SPACES, AND THE INJECTIVE ENVELOPE

David P. Blecher and Vern I. Paulsen 


\title{
MULTIPLIERS OF OPERATOR SPACES, AND THE INJECTIVE ENVELOPE
}

\author{
David P. Blecher and Vern I. PAulsen
}

\begin{abstract}
We study the injective envelope $I(X)$ of an operator space $X$, showing amongst other things that it is a self-dual $C^{*}$ module. We describe the diagonal corners of the injective envelope of the canonical operator system associated with $X$. We prove that if $X$ is an operator $A$ - $B$-bimodule, then $A$ and $B$ can be represented completely contractively as subalgebras of these corners. Thus, the operator algebras that can act on $X$ are determined by these corners of $I(X)$ and consequently bimodule actions on $X$ extend naturally to actions on $I(X)$. These results give another characterization of the multiplier algebra of an operator space, which was introduced by the first author, and a short proof of a recent characterization of operator modules, and a related result. As another application, we extend Wittstock's module map extension theorem, by showing that an operator $A$-B-bimodule is injective as an operator $A$ - $B$-bimodule if and only if it injective as an operator space.
\end{abstract}

\section{Introduction.}

In this paper we investigate some connections between the following topics: Injectivity of operator spaces, self-dual Hilbert $\mathrm{C}^{*}$-modules (in the sense of Paschke); completely contractive actions of one operator space on another; and the notion of a multiplier operator algebra of an operator space which was recently introduced by the first author.

The results and definitions follow a natural logical sequence, so we begin without further delay. We refer to [7] for additional information, and complementary results, and to $[\mathbf{2 9}, \mathbf{1 5}, \mathbf{3 2}]$ for background information on operator spaces and completely bounded maps.

Recall that an operator space $X$ is injective, if for any operator spaces $W \subset Z$, and any completely bounded linear $T: W \rightarrow X$, there exists a linear $\widetilde{T}: Z \rightarrow X$ extending $T$, with $\|T\|_{c b}=\|\widetilde{T}\|_{c b}$. It has been known for a long time that $B(H)$ is an injective operator space (see $[\mathbf{2 9}, \mathbf{3 9}, \mathbf{2 8}, \mathbf{2}]$ ) for any Hilbert space $H$. In 1983, Youngson showed [40] that an injective operator space $X$ is a 'corner' of a $\mathrm{C}^{*}$-algebra $A$, by which we mean that there exist 
projections $p, q$ in the multiplier algebra $\mathcal{M}(A)$ of $A$, such that $X=p A q$. It is well-known [34] that this last condition is equivalent to saying that such an $X$ is a Hilbert $C^{*}$-module. Then Hamana in 1985 (see notes in $[\mathbf{1 9}, \mathbf{2 0}])$, and Ruan independently $[\mathbf{3 5}]$, showed that any operator space $X$ has an operator space injective envelope $I(X)$. To prove the existence of this envelope, one may follow the classical construction for Banach space injective envelopes. We will sketch the main idea: One begins by choosing any injective object $B$ containing $X$. Then one considers the $X$-projections on $B$, by which we mean completely contractive idempotent maps on $B$ which fix $X$. An idempotent map of course is one such that $\Psi \circ \Psi=\Psi$. There is a natural ordering on such maps, and, with a little work one can show, by a Zorn's lemma argument, that there is a minimal $X$-projection $\Phi$. The range of $\Phi$ in $B$ may be taken to be the injective envelope $I(X)$ of $X$, and one has $X \subset I(X) \subset B$. Thus one sees that $I(X)$ is the smallest injective space containing $X$. As in the Banach space case (see $[\mathbf{2 1}$, Section 11] for details and references), one proves that $I(X)$ is an 'essential' and 'rigid' extension of $X$. The latter term, rigidity, means that the identity map is the only completely contractive map on $I(X)$ extending the identity map on $X$.

Note that if $\mathcal{S}$ is a linear subspace of $B(H)$ containing $I_{H}$ (for example, if $\mathcal{S}$ is a unital $\mathrm{C}^{*}$-algebra), then one may choose $B=B(H)$ in the above. Since $\Phi(I)=I$, it follows that $\Phi$ is completely positive [29]. A well-known theorem of Choi and Effros [12] states that the range of a completely positive unital idempotent map on a $\mathrm{C}^{*}$-algebra, is a $\mathrm{C}^{*}$-algebra with respect to a certain multiplication. Hence it follows that $I(\mathcal{S})$ is a unital $\mathrm{C}^{*}$-algebra. ${ }^{1}$

Hamana also gives another construction of $I(X)$ to the one outlined above, which allows one to prove something a little stronger. Since this construction will be important for us, we will outline some of the ideas. By the method popularized by the second author (see [29] Lemma 7.1), we may embed the operator space $X$ in a canonical unital operator system ${ }^{2}$

$$
\mathcal{S}(X)=\left(\begin{array}{cc}
\mathbb{C} & X \\
X^{*} & \mathbb{C}
\end{array}\right)
$$

If one forms the injective envelope $I(\mathcal{S}(X))$, it will be a unital $\mathrm{C}^{*}$-algebra, by the aforementioned argument using the Choi-Effros result. Indeed, since the minimal $\mathcal{S}(X)$-projection fixes the $\mathrm{C}^{*}$-algebra $\mathbb{C} \oplus \mathbb{C}$ which is the diagonal of $\mathcal{S}(X)$, it follows immediately (for example by Lemma 1.6 below, although this is not necessary), that the following elements of $\mathcal{S}(X)$ are two selfadjoint

\footnotetext{
${ }^{1} \mathrm{In}$ fact, $I(A)$ is a unital $\mathrm{C}^{*}$-algebra even for a non-unital $\mathrm{C}^{*}$-algebra $A$. Since we have no good reference for this no doubt well known fact, we supply a proof later.

${ }^{2}$ An operator system is a selfadjoint linear subspace of $B(H)$ containing $I_{H}$.
} 
projections with sum 1 in the $\mathrm{C}^{*}$-algebra $I(\mathcal{S}(X))$ :

$$
p=\left(\begin{array}{cc}
1 & 0 \\
0 & 0
\end{array}\right), \quad q=\left(\begin{array}{cc}
0 & 0 \\
0 & 1
\end{array}\right) .
$$

Therefore, with respect to $p$ and $q$, we may decompose $I(\mathcal{S}(X))$ to write it as consisting of $2 \times 2$ matrices. Hamana shows that $p I(\mathcal{S}(X)) q$, the 1-2 corner of $I(\mathcal{S}(X))$, is the injective envelope of $X$. This recovers and strengthens Youngson's result. The four corners of $I(\mathcal{S}(X))$ we will name:

$$
I(\mathcal{S}(X))=\left(\begin{array}{cc}
I_{11} & I(X) \\
I(X)^{*} & I_{22}
\end{array}\right) .
$$

It is clear that $I_{11}$ and $I_{22}$ are also injective $\mathrm{C}^{*}$-algebras.

We will write $j$ for the canonical inclusion of $X$ inside $I(X)$.

Writing $I=I(X)$ for a moment, we define a subset of $I(\mathcal{S}(X))$ by

$$
\mathcal{L}(I(X))=\left(\begin{array}{cc}
I I^{*} & I \\
I^{*} & I^{*} I
\end{array}\right)
$$

where $I I^{*}$ for example, is the closed span in $I_{11}$ of terms $x y^{*}$ with $x, y \in I$. Constructions related to this one have been studied by Hamana, Ruan, and C. Zhang ([41]). Henceforth, we will reserve the letters $\mathcal{C}(X)$ and $\mathcal{D}(X)$ for $I I^{*}$ and $I^{*} I$ respectively. When $X$ is understood we will simply write $\mathcal{C}$ and $\mathcal{D}$. Thus $\mathcal{C} \subset I_{11}, \mathcal{D} \subset I_{22}$ as $\mathrm{C}^{*}$-subalgebras. Notice also that $\mathcal{L}(I(X))$ coincides with the smallest closed 2-sided ideal in $I(\mathcal{S}(X))$ containing the copy of $I(X)$ in the 1-2-corner of $I(\mathcal{S}(X))$, and this fact will be used below.

Clearly $\mathcal{L}(I(X))$ is a $\mathrm{C}^{*}$-subalgebra of $I(\mathcal{S}(X))$. From this, or otherwise, it is easy to see that $I(X)$ is a $\mathcal{C}-\mathcal{D}$-bimodule which, with respect to the natural $\mathcal{C}$ - and $\mathcal{D}$-valued inner products given by $x y^{*}$ and $x^{*} y$, is a 'strong Morita equivalence $\mathcal{C}-\mathcal{D}$-bimodule' in the language of Rieffel. Sometimes this is also referred to as a ' $\mathcal{C}-\mathcal{D}$-imprimitivity bimodule'. It follows by basic $\mathrm{C}^{*}$-algebraic Morita theory (see $[\mathbf{3 4}, \mathbf{2 2}]$ say), that $\mathcal{C} \cong \mathbb{K}(I(X))$ as $\mathrm{C}^{*}$-algebras, where $\mathbb{K}(I(X))$ is the so called 'imprimitivity $\mathrm{C}^{*}$-algebra' of the right $\mathcal{D}$-module $I(X)$. Also, $\mathcal{M}(\mathcal{C}) \cong \mathbb{B}_{\mathcal{D}}(I(X))$, the adjointable $\mathcal{D}$-module maps on $I(X)$. In fact we shall see that the left multiplier algebra $L M(\mathcal{C})$ (which by a result of Lin [24] may be identified with the space $B_{\mathcal{D}}(I(X))$ of bounded right $\mathcal{D}$-module maps on $I(X)$ ) coincides with $\mathcal{M}(\mathcal{C})$. Also $\mathcal{L}(I(X))$ may be identified with the 'linking $\mathrm{C}^{*}$-algebra' [34] of the bimodule $I(X)$. Although this will not be explicitly used below, it is a useful perspective.

We shall write $\mathcal{S}_{0}(X)$ for the subspace of $\mathcal{S}(X)$ consisting of those elements with 0 's on the main diagonal.

Proposition 1.1. For any operator space $X$, we have that

$$
J=I(\mathcal{S}(X)) \mathcal{S}_{0}(X) I(\mathcal{S}(X))
$$

is an essential ideal in $I(\mathcal{S}(X))$. 
Proof. Suppose that $K$ is an ideal in $I(\mathcal{S}(X))$ whose intersection with $J$ is zero. Let $\pi: I(\mathcal{S}(X)) \rightarrow I(\mathcal{S}(X)) / K$. Thinking of $I(\mathcal{S}(X))$ as $2 \times 2$ matrices, it is clear that $\pi$ maps each of the 4 corners into a matching 'corner' of $I(\mathcal{S}(X)) / K$. Since $\pi$ is $1-1$ on $J$, it is completely isometric on $\mathcal{S}_{0}(X)$. Let $\Phi$ be the restriction of $\pi$ to $\mathcal{S}(X)$. By Lemma 7.1 in [29], $\Phi$ is a complete order injection.

Extend the map from $\pi(\mathcal{S}(X)) \rightarrow \mathcal{S}(X)$ which is the inverse of $\Phi$, to a $\operatorname{map} \gamma: I(\mathcal{S}(X)) / K \rightarrow I(\mathcal{S}(X))$. Since $\gamma \circ \Phi=I d_{\mathcal{S}(X)}$, it follows by rigidity that $\gamma \circ \pi=I d_{I(\mathcal{S}(X))}$. Thus $K=(0)$.

Corollary 1.2. For any operator space $X$, we have that $\mathcal{L}(I(X))$ is an essential ideal in $I(\mathcal{S}(X))$, and that $\mathcal{C}=\mathcal{C}(X)$ is an essential ideal in $I_{11}$. Thus we have the following canonical inclusions of $C^{*}$-algebras

$$
\mathcal{C} \subset I_{11} \subset M(\mathcal{C})
$$

If $\mathcal{C}$ is represented faithfully and nondegenerately on a Hilbert space $H$, then this string may be regarded as inclusions of subalgebras of $B(H)$. Similar assertions hold for $\mathcal{D}(X) \subset I_{22}$.

Proof. Clearly $J \subset \mathcal{L}(I(X)) \subset I(\mathcal{S}(X))$, where $J$ is as in Proposition 1.1. Thus $\mathcal{L}(I(X))$ is an essential ideal in $I(\mathcal{S}(X))$, by that Proposition. To see the second assertion, notice that if $t \in I_{11}$ and $t \mathcal{C}=0$, then $t \mathcal{C} t^{*}=0$, which implies that $t z z^{*} t^{*}=t z=0$ for all $z \in I(X)$. It follows immediately that $(t \oplus 0) \mathcal{L}(I(X))=0$. Hence by the first assertion, $t=0$. Thus $\mathcal{C}$ is an essential ideal in $I_{11}$.

That $I_{11} \subset M(\mathcal{C})$ follows from the universal property of the multiplier algebra, namely that $M(\mathcal{C})$ contains a copy of any $\mathrm{C}^{*}$-algebra containing $\mathcal{C}$ as an essential ideal ([22] Chapter 2, say).

Since $\mathcal{C}$ is essential in $I_{11}$, it follows from [16] Theorem 4.5, that $M(\mathcal{C}) \subset$ $I_{11}$. Thus in fact $I_{11}=M(\mathcal{C}) \cong \mathbb{B}_{\mathcal{D}}(I(X))$. However we will deduce all this in a self-contained way, from some machinery we develop next:

Corollary 1.3. If $t \in I_{11}$, and if $t x=0$ for all $x \in X$, then $t=0$.

Proof. Without loss of generality, we may assume that $\|t\| \leq 1$. By replacing $t$ by $t^{*} t$, we may also suppose that $0 \leq t \leq 1$, where this last ' 1 ' is the identity of $I_{11}$. Let $p=1-t$. Define $\phi(z)=p z$, for $z \in I(X)$. Since $\phi(x)=x$ for $x \in X$, we obtain $\phi=I d$, by rigidity. Thus $t I(X)=0$, which (we showed in the proof of Corollary 1.2) implies that $t=0$.

Definition 1.4. Let $X$ be an operator space. We define the left multiplier operator algebra of $X$ to be $I M_{l}(X)=\left\{T \in I_{11}: T X \subset X\right\}$. We define the left multiplier $C^{*}$-algebra of $X$ to be $I M_{l}^{*}(X)=\left\{T \in I M_{l}(X): T^{*} \in\right.$ $\left.I M_{l}(X)\right\}$. 
Note that $I M_{l}^{*}(X)$ is a $\mathrm{C}^{*}$-algebra, whereas $I M_{l}(X)$ is a unital nonselfadjoint operator algebra in general. There is a similar definition for right multipliers.

We shall soon see that these multiplier algebras coincide with the ones introduced in [7] $\S 4$. These simultaneously generalize the common operator algebras associated with Hilbert $\mathrm{C}^{*}$-modules, the multiplier function algebras of a Banach space introduced by Alfsen and Effros [1] (who only considered the real scalar case - see [4] for the complex case), and the multiplier algebras of a nonselfadjoint operator algebra with c.a.i..

Definition 1.5. Let $H, K$ be Hilbert spaces. A multiplication situation on $H \oplus K$ consists of three concrete operator spaces $X, Y, Z$ such that $Y \subset$ $B(H), X \subset B(K, H)$, and $Z \subset B(K)$, and such that $Y X \subset X$ and $X Z \subset$ $X$.

We will need the following elementary lemma (c.f. [29] Ex. 4.2-4.5):

Lemma 1.6 (Choi [11]). Suppose that $\phi: A \rightarrow B$ is a completely positive map between $C^{*}$-algebras, with $\phi(1)=1$. Suppose that there is a $C^{*}$ subalgebra $N$ of $A$ with $1_{A} \in N$, such that $\pi=\phi_{\left.\right|_{N}}$ is a ${ }^{*}$-homomorphism. Then $\phi$ is an ' $N$-bimodule map'. That is,

$$
\phi(a n)=\phi(a) \pi(n) \quad \text { and } \quad \phi(n a)=\pi(n) \phi(a)
$$

for all $a \in A, n \in N$.

We will refer to the following as the 'multiplication theorem':

Theorem 1.7. $\quad$ (i) Suppose that $X$ is an operator space, and that $I_{11}, I_{22}$ are the diagonal corners of $I(\mathcal{S}(X))$, as usual. If $Y, Z$ are two operator spaces such that $X, Y, Z$ form a multiplication situation on $H \oplus K$, as above, then there exist unique completely contractive linear maps $\theta: Y \rightarrow I M_{l}(X)$ and $\pi: Z \rightarrow I M_{r}(X)$ such that $\theta(y) j(x)=j(y x)$, and $j(x) \pi(z)=j(x z)$, for all $x \in X, y \in Y, z \in Z$.

(ii) If in addition, $Y$ is a subalgebra (resp. *-subalgebra) of $B(H)$, then $\theta$ is also a homomomorphism (resp. ${ }^{*}$-homomomorphism into $I M_{l}^{*}(X)$ ). Similarly for $Z$.

Proof. By [29] Lemma 7.1, we have a completely order isomorphic copy $\mathcal{G}$ of $\mathcal{S}(X)$ inside $B(H \oplus K)$. By [17] Corollary 4.2, there exists a surjective *-homomorphism from the $\mathrm{C}^{*}$-subalgebra $C^{*}(\mathcal{G})$ of $B(H \oplus K)$ generated by $\mathcal{G}$, onto the $\mathrm{C}^{*}$-envelope $C_{e}^{*}(\mathcal{S}(X))$, which fixes the copies of $\mathcal{S}(X)$. Let $\phi: B(H \oplus K) \rightarrow I(\mathcal{S}(X))$ be a completely positive map extending the *homomorphism. Since $\phi$ fixes the diagonal scalars $\mathbb{C} \oplus \mathbb{C}$, it follows by 1.6 (this is a common argument), that $\phi$ decomposes as a $2 \times 2$ matrix of maps, each corner map defined on the corresponding 'corner' of $B(H \oplus K)$. In 
particular, we have

$$
\phi\left(\left[\begin{array}{ll}
y & x \\
0 & z
\end{array}\right]\right)=\left[\begin{array}{cc}
\theta(y) & j(x) \\
0 & \pi(z)
\end{array}\right]
$$

for a map $\theta: Y \rightarrow I_{11}, \pi: Z \rightarrow I_{22}$, and for all $x \in X, y \in Y, z \in Z$. By the previous lemma, $\phi$ is a ' $C^{*}(\mathcal{G})$-bimodule map'. Hence for $y \in Y, x \in X$ we have:

$$
\begin{aligned}
{\left[\begin{array}{cc}
0 & j(y x) \\
0 & 0
\end{array}\right] } & =\phi\left(\left[\begin{array}{ll}
y & 0 \\
0 & 0
\end{array}\right]\left[\begin{array}{ll}
0 & x \\
0 & 0
\end{array}\right]\right) \\
& =\phi\left(\left[\begin{array}{ll}
y & 0 \\
0 & 0
\end{array}\right]\right)\left[\begin{array}{cc}
0 & j x \\
0 & 0
\end{array}\right]=\left[\begin{array}{cc}
\theta(y) & 0 \\
0 & 0
\end{array}\right]\left[\begin{array}{cc}
0 & j x \\
0 & 0
\end{array}\right] .
\end{aligned}
$$

Thus $j(y x)=\theta(y) j(x)$. The uniqueness of $\theta$ follows from Corollary 1.3. Similarly for $\pi$.

For (ii), note that for $y_{1}, y_{2} \in Y$ and $x \in X$ we have $\left(y_{1} y_{2}\right) x=\theta\left(y_{1} y_{2}\right) x=$ $\theta\left(y_{1}\right) \theta\left(y_{2}\right) x$. Now use Corollary 1.3 to conclude that $\theta$ is a homomorphism. The last assertion follows from the fact that a contractive representation of a $\mathrm{C}^{*}$-algebra is a ${ }^{*}$-homomorphism.

We recall that a right $\mathrm{C}^{*}$-module $Z$ over $D$ is 'self-dual', if it satisfies the equivalent of the Riesz representation theorem for Hilbert spaces, namely that every $f \in B_{D}(Z, D)$ is given by the inner product with a fixed $z \in Z$.

Corollary 1.8. If $X$ is an operator space, then:

(i) $I_{11}=M(\mathcal{C}(X))=\operatorname{LM}(\mathcal{C}(X))=R M(\mathcal{C}(X))=Q M(\mathcal{C}(X))=I(\mathcal{C}(X))$. Thus $I_{11} \cong \mathbb{B}_{\mathcal{D}}(I(X))=B_{\mathcal{D}}(I(X))$. Similarly, $I_{22}=M(\mathcal{D})=L M(\mathcal{D})$ $=I(\mathcal{D})$.

(ii) $I(X)$ is a self-dual right $C^{*}$-module (over $\mathcal{D}$ or over $\mathcal{M}(\mathcal{D})$ ). Similarly, it is a self-dual left $C^{*}$-module.

(iii) $I(\mathcal{S}(X))$ is the multiplier $C^{*}$-algebra of $\mathcal{L}(I(X))$. Also, $I(\mathcal{S}(X))$ is the injective envelope of $\mathcal{L}(I(X))$.

Proof. (i): Represent $\mathcal{L}(I(X))$ non-degenerately on a Hilbert space $H \oplus K$. We obtain a multiplication situation on $H \oplus K$ given by the actions of $L M(\mathcal{C})$ and $R M(\mathcal{D})$ on $I(X)$. By the multiplication theorem, we get a completely contractive homomomorphism $\theta: L M(\mathcal{C}) \rightarrow I_{11}$ such that $\theta(T) x=T x$, for all $x \in I(X)$ and $T \in L M(\mathcal{C})$. Hence $T=\theta(T) \in I_{11}$. Thus by Corollary 1.2, we have $I_{11}=M(\mathcal{C}(X))=L M(\mathcal{C}(X))$. By Lin's theorem [24], the latter $\mathrm{C}^{*}$-algebra may be identified with $B_{\mathcal{D}}(I(X))$. Taking adjoints gives $R M(\mathcal{C}(X))=M(\mathcal{C}(X))$. As noted in [25], (ii) below implies that $M(\mathcal{C}(X))=Q M(\mathcal{C}(X))$. Finally, note that we have the following $\mathrm{C}^{*}$ subalgebras: $\mathcal{C} \subset I_{11}=M(\mathcal{C}) \subset I(\mathcal{C})$. The last inclusion is a fact from [16] which is reproved at the end of our paper. By the injectivity of $I_{11}$ there 
exists a completely contractive projection $\Phi$ from $I(\mathcal{C})$ onto $I_{11}$; since $\Phi$ fixes $\mathcal{C}$ we see by rigidity that $\Phi=I d$ and $I_{11}=I(\mathcal{C})$.

(ii): We apply (i) but with $X$ replaced by the right $\mathrm{C}^{*}$-module sum $I(X) \oplus_{c} M(\mathcal{D})$. Clearly the latter is a full $\mathrm{C}^{*}$-module over $M(\mathcal{D})$, and it is injective since it is a corner of $I(\mathcal{S}(X))$. Using (i) and Corollary A.7 in [7] if necessary, we obtain that $\mathbb{B}_{M(\mathcal{D})}\left(I(X) \oplus_{c} M(\mathcal{D})\right)=B_{M(\mathcal{D})}\left(I(X) \oplus_{c} M(\mathcal{D})\right)$, from which it is easy to see that $I(X)$ is a self-dual $M(\mathcal{D})$-module. However $B_{\mathcal{D}}(I(X), \mathcal{D})=B_{M(\mathcal{D})}(I(X), M(\mathcal{D}))$ by Cohen's factorization theorem.

(iii): It is well-known that for any full right $\mathrm{C}^{*}$-module $Z$ over a $\mathrm{C}^{*}$-algebra $B$ say, we have that $\mathbb{K}_{\mathcal{D}}\left(Z \oplus_{c} \mathcal{D}\right)$ is a copy of the linking $\mathrm{C}^{*}$-algebra of $Z$. Also $\mathbb{B}_{\mathcal{D}}\left(Z \oplus_{c} \mathcal{D}\right)=M\left(\mathbb{K}_{\mathcal{D}}\left(Z \oplus_{c} \mathcal{D}\right)\right)$. Thus we have $M(\mathcal{L}(I(X)))=\mathbb{B}_{\mathcal{D}}(I(X) \oplus \mathcal{D})$. This of course splits into four corners. The 1-1 corner is $\mathbb{B}_{\mathcal{D}}(I(X))=I_{11}$ by (i). The $2-1$ corner is $\mathbb{B}_{\mathcal{D}}(I(X), \mathcal{D}) \cong I(X)^{*}$ by (ii). The $2-2$ corner is $\mathbb{B}_{\mathcal{D}}(\mathcal{D}, \mathcal{D})=M(\mathcal{D})=I_{22}$ by (i). This gives the first result. The second follows just as $I(\mathcal{C})=I_{11}$ in (i).

Theorem 1.9. If $X$ is an operator space, then:

(i) $I M_{l}(X)\left(\right.$ resp. $\left.I M_{l}^{*}(X)\right)$ is completely isometrically isomorphic (resp.

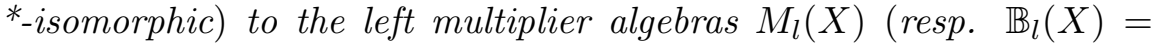
$\left.A_{l}(X)\right)$ defined in $[\mathbf{7}$, Section 4$]$.

(ii) $I M_{l}^{*}(X)$ is isometrically isomorphic to a closed subalgebra of $B(X)$. Also, $I M_{l}^{*}(X)$ is completely isometrically isomorphic to a closed subalgebra of $B_{l}(X)$ or $C B_{l}(X)$.

Before we prove this, we define $B_{l}(X)$ and $C B_{l}(X)$ for an operator space $X$. Namely $B_{l}(X)=B(X)$ but with matrix norms

$$
\left\|\left[T_{i j}\right]\right\|_{n}^{l}=\sup \left\{\left\|\left[\sum_{k=1}^{n} T_{i k}\left(x_{k}\right)\right]\right\|_{C_{n}(X)}: x \in \operatorname{BALL}\left(C_{n}(X)\right)\right\} .
$$

(Here $C_{n}(X)=X^{n}$, but with the operator space structure one gets by identifying $C_{n}(X)$ with the 'first column' of the operator space $M_{n}(X)$.) With these norms $B_{l}(X)$ is not a matrix normed space in the traditional sense. However $M_{n}\left(B_{l}(X)\right)$ is a unital Banach algebra. Similarly one defines $C B_{l}(X)$, the only difference being that one replaces $x_{k}$ in the expression above by $x_{(k, p), q}$. Here $\left[x_{(k, p), q}\right]$ is a matrix indexed on rows by $(k, p)$ and on columns by $q$. Again $M_{n}\left(C B_{l}(X)\right)$ is a unital Banach algebra.

Proof. (i): By 1.8, any $T \in I M_{l}(X)$ may be viewed as a bounded, and hence completely bounded, module map on $I(X)$. We obtain a canonical sequence of completely contractive homomorphisms

$$
I M_{l}(X) \stackrel{\rho}{\rightarrow} M_{l}(X) \rightarrow C B(X)
$$

given by restriction of domain. By Corollary 1.3, these homomorphisms are $1-1$. 
On the other hand, we have a multiplication situation given by the action of $M_{l}(X)$ on $X$. Hence, by the multiplication theorem, there exists a completely contractive homomorphism $\theta: M_{l}(X) \rightarrow I_{11}$ such that $\theta(T) x=T x$ for all $T \in M_{l}(X), x \in X$. Thus $\rho \theta=I d$. Thus $\rho$ is onto, and since $\rho$ is $1-1$ we obtain that $I M_{l}(X) \cong M_{l}(X)$ completely isometrically and as operator algebras.

(ii) The first statement follows from a result which may be found in [37] Proposition 1.1 or [5] Corollary 1, which asserts that any contractive homomorphism from a $\mathrm{C}^{*}$-algebra into a Banach algebra, is a $*$-homomorphism onto its range, which is a $\mathrm{C}^{*}$-algebra (with the norm and algebra structure inherited from the Banach algebra). Hence the canonical homomorphism $\mathbb{B}_{l}(X) \rightarrow C B(X) \quad\left(\right.$ or $\left.\mathbb{B}_{l}(X) \rightarrow B(X)\right)$ is a $*$-homomorphism onto a $\mathrm{C}^{*}$ algebra. Since the homomorphism is 1-1 it is therefore isometric.

The second statement follows by considering the following canonical isometric inclusions

$$
M_{n}\left(\mathbb{B}_{l}(X)\right) \subset M_{n}\left(\mathbb{B}_{\mathcal{D}}(I(X))\right) \subset M_{n}\left(B_{l}(I(X))\right),
$$

the last inclusion following from Section 3 of [26]. Thus by restriction of domain, we get a contractive unital 1-1 homomorphism $M_{n}\left(\mathbb{B}_{l}(X)\right) \rightarrow$ $M_{n}\left(B_{l}(X)\right)$. Now we can apply [5] Corollary 1 to deduce that this last homomorphism is an isometry.

A similar argument works for $C B_{l}$.

It follows from (i) and a result in [7, Section 4], that the subalgebra of $B(X)$ or $C B(X)$ corresponding to $I M_{l}^{*}(X)$ by (ii) above, is the $\mathrm{C}^{*}$-algebra of (left) adjointable operators $\mathcal{A}_{l}(X)$ on $X$.

We do not know whether $I M_{l}^{*}(X)$ is completely isometrically contained inside $C B(X)$ in general. However it is not hard to find examples showing that the canonical contraction $M_{l}(X) \rightarrow B(X)$ (or into $C B(X)$ ) is not an isometry in general (see [7]). This shows that if $T \in \mathbb{B}_{\mathcal{D}}(I(X))$, with $T(X) \subset X$, then one cannot expect anything like $\|T\|_{c b}=\left\|T_{\left.\right|_{X}}\right\|_{c b}$.

Finally we remark that if $I M_{l}(X)=\mathbb{C}$, then it follows from the multiplication theorem that for any linear complete isometry $i: X \rightarrow B(K, H)$ such that $\left[i(X) K\left\lceil=H\right.\right.$, we have that scalar multiples of $I_{H}$ are the only operators $T \in B(H)$ such that $T i(X) \subset i(X)$.

\section{Applications.}

We recall from [7] that an oplication of an operator space $Y$ on an operator space $X$, is a bilinear map o: $Y \times X \rightarrow X$, such that

(1) $\|y \circ x\|_{n} \leq\|y\|_{n}\|x\|_{n}$, for all $n \in \mathbb{N}, x \in M_{n}(X), y \in M_{n}(Y)$,

(2) there is an element $e \in Y_{1}$ such that $e \circ x=x$ for all $x \in X$. 
In (1), $y \circ x$ is computed by the usual rule for multiplying matrices.

We will apply the multiplication theorem from $\S 1$ to give a proof of the 'oplication theorem' from [7] §5:

Theorem 2.1. Suppose that $Y, X$ are operator spaces, and suppose that $\circ: Y \times X \rightarrow X$ is an oplication, with 'identity' $e \in Y$. Then there exists a unique completely contractive linear map $\theta: Y \rightarrow M_{l}(X)$ such that $y \circ x=$ $\theta(y) x$, for all $y \in Y, x \in X$. Also $\theta(e)=1$. Moreover, if $Y$ is, in addition, an algebra with identity $e$, then $\theta$ is a homomorphism if and only if $\circ$ is a module action. On the other hand, if $Y$ is a $C^{*}$-algebra (or operator system) with identity $e$, then $\theta$ has range inside $\mathbb{B}_{l}(X)$, and is completely positive and *-linear.

Proof. As in [7] the difficult part is to prove the first statement, the existence of $\theta$. (The uniqueness follows from 1.3.) Indeed it suffices to find Hilbert spaces $H, K$, a complete isometry $\Phi: X \rightarrow B(K, H)$, and a linear complete contraction $\theta: Y \rightarrow B(H)$, such that $\theta(e)=I$, and such that $\Phi(y \circ x)=$ $\theta(y) \Phi(x)$, for all $y \in Y, x \in X$. For in that case $\theta(Y), \Phi(X), \mathbb{C}$ would form a multiplication situation on $H \oplus K$. Now use the multiplication theorem above, together with the fact that $I M_{l}(X) \cong M_{l}(X)$, to obtain the existence of $\theta$.

The existence of such $\Phi$ etc., follows easily from Le Merdy's proof of the 'BRS' theorem ([23] 3.3). Namely, first suppose that $X \subset B(K)$. By the 'multilinear Stinespring' theorem of $[\mathbf{1 4}, \mathbf{3 0}]$, we may write $y \circ x=$ $\beta_{1}(y) \alpha_{1}(x)$, for completely contractive maps $\alpha_{1}: X \rightarrow B\left(K, H_{1}\right)$ and $\beta_{1}$ : $Y \rightarrow B\left(H_{1}, K\right)$. Similarly, $\alpha_{1}(y \circ x)=\beta_{2}(y) \alpha_{2}(x)$, where now $\alpha_{2}: X \rightarrow$ $B\left(K, H_{2}\right)$ say. Inductively we obtain, $\alpha_{k-1}(y \circ x)=\beta_{k}(y) \alpha_{k}(x)$, where $\alpha_{k}: X \rightarrow B\left(K, H_{k}\right)$, say. Since $e \circ x=x$, we see that each $\alpha_{k}$ is a complete isometry. Let $W=X \otimes K$, and define $f_{k}: W \rightarrow H_{k}$ by $f_{k}(x \otimes \zeta)=\alpha_{k}(x)(\zeta)$. It is easy to check that $\left\|f_{k}(w)\right\|_{H_{k}} \leq\left\|f_{k+1}(w)\right\|_{H_{k+1}}$, for $w \in W$, as in [23]. Hence (by the parallelogram law if necessary) it is clear that $\lim _{k}\left\|f_{k}(\cdot)\right\|_{H_{k}}$ defines a seminorm which gives rise to a Hilbert space norm (on the quotient of $W$ by the nullspace of the seminorm). Write this resulting Hilbert space as $H$. There is an obvious map $\theta: Y \rightarrow L(H)$, given by $\theta(y)([x \otimes \zeta])=$ $[(y \circ x) \otimes \zeta]$. It is easy to see that this is completely contractive as in [23]. The map $\Phi: X \rightarrow B(K, H)$ given by $\Phi(x)(\zeta)=[x \otimes \zeta]$ is clearly a complete contraction, too. On the other hand, for any $\zeta \in \operatorname{Ball}(K)$, we have

$$
\|\Phi(x)\| \geq\|\Phi(x)(\zeta)\|=\lim _{k}\left\|\alpha_{k}(x)(\zeta)\right\| \geq\left\|\alpha_{1}(x)(\zeta)\right\| .
$$

Thus $\|\Phi(x)\| \geq\left\|\alpha_{1}(x)\right\|=\|x\|$, showing that $\Phi$ is an isometry. A similar argument shows that $\Phi$ is a complete isometry.

As shown in [7], this theorem has very many consequences, containing as special cases, the 'BRS' theorem [10], and many other results. The original 
proof of the above theorem in [7] was much more difficult. The first author has subsequently replaced that proof there with another, part of which is similar to the proof above, but avoids use of the 'multiplication theorem' above. The version in [7] also does not give the fact that $I M_{l}(X)=M_{l}(X)$.

A left operator module $X$ over a unital operator algebra $A$, is an operator space which is also a unitary left $A$-module (unitary means that $1 x=x$ for all $x \in X)$, such that $\|a x\| \leq\|a\|\|x\|$ for all matrices $a$ with entries in $A$ and $x$ with entries in $X$. A similar definition holds for right modules or bimodules.

One may deduce from the oplication theorem the following refinement of the Christensen-Effros-Sinclair representation theorem for operator modules [13]. We will in fact give an independent proof (which is the essentially the same as the proof above, except that we use the original Christensen-EffrosSinclair theorem (which is quite simple) instead of the Le Merdy argument). In any case note that the method shows that the $A$-B-action on an operator $A$ - $B$-bimodule $X$, may be extended to an action on $I(X)$, making $I(X)$ an operator $A$ - $B$-bimodule.

Theorem 2.2. Suppose that $A$ and $B$ are unital operator algebras, and that $X$ is an operator space and a unitary $A$-B-bimodule with respect to a bimodule action $m: A \times X \times B \rightarrow X$. The following are equivalent:

(i) $X$ is an operator $A$-B-bimodule.

(ii) There exist Hilbert spaces $H$ and $K$, and a linear complete isometry $\Phi: X \rightarrow B(K, H)$ and completely contractive unital homomorphisms $\theta: A \rightarrow B(H)$ and $\pi: B \rightarrow B(K)$, such that $\Phi(m(a, x, b))=$ $\theta(a) \Phi(x) \pi(b)$, for all $a \in A, x \in X$ and $b \in B$.

(iii) There exists unique completely contractive unital homomorphisms $\theta$ : $A \rightarrow M_{l}(X)$ and $\pi: B \rightarrow M_{r}(X)$ such that $\theta(a) x \pi(b)=m(a, x, b)$ for all $a \in A, x \in X$ and $b \in B$.

Proof. That (i) is equivalent to (ii) is a restatement of the ChristensenEffros-Sinclair representation theorem.

(iii) $\Longrightarrow$ (ii): Obvious.

(ii) $\Longrightarrow$ (iii): $\theta(A), \Phi(X), \pi(B)$ form a multiplication situation on $H \oplus K$. The result then follows by the multiplication theorem.

We will refer to a triple $(\Phi, \theta, \pi)$ as in (ii) above as a CES representation of the bimodule $X$. We will call it a faithful CES representation if $\theta$ and $\pi$ are also completely isometric. It is always possible, by an obvious direct sum trick, to find a faithful CES representation for an operator bimodule. For any CES representation of $X$ we obtain an 'upper triangular $2 \times 2$ operator algebra', namely

$$
\mathcal{U}(X)=\left[\begin{array}{cc}
\theta(A) & \Phi(X) \\
0 & \pi(B)
\end{array}\right]
$$


We will write $\mathcal{U}_{e}(X)$ for this algebra in the case that $\left(j, \theta_{e}, \pi_{e}\right)$ is the representation in (iii) above, into the multiplier algebras. Thus $\mathcal{U}_{e}(X) \subset I(\mathcal{S}(X))$.

If $A$ and $B$ are $\mathrm{C}^{*}$-algebras, and if we take faithful CES representations and form $\mathcal{U}(X)$, then it is clear from $[36]$ that $\mathcal{U}(X)$ as an abstract operator algebra (i.e., as an algebra and an operator space), is independent of the particular faithful CES representation. This is probably not true if $A, B$ are non-selfadjoint. However in either case, we can easily see that the triple $\left(j, \theta_{e}, \pi_{e}\right)$ given in (iii) above, is the 'smallest' CES representation of $X$ :

Corollary 2.3. Suppose that $A$ and $B$ are unital operator algebras, and that $X$ is an $A$-B-operator bimodule. Let $(\Phi, \theta, \pi)$ be a CES representation of $X$, and let $\mathcal{U}(X)$ be the corresponding upper triangular $2 \times 2$ operator algebra. Then there is a canonical completely contractive unital homomorphism $\phi$ : $\mathcal{U}(X) \rightarrow \mathcal{U}_{e}(X)$, which takes each corner of $\mathcal{U}(X)$ into the same corner for $\mathcal{U}_{e}(X)$. Indeed $\phi$ induces completely contractive unital homomorphisms $\rho$ and $\sigma$, from $\theta(A)$ and $\pi(B)$, to $\theta_{e}(A)$ and $\pi_{e}(B)$ respectively, such that $\rho \circ \theta=\theta_{e}$ and $\sigma \circ \pi=\pi_{e}$.

Proof. This follows immediately from the proof of Theorem 1.7, and of the implication '(ii) $\Longrightarrow$ (iii)' of 2.2. The $\phi$ is as in the proof of Theorem 1.7, which is easily seen to be a homomorphism on $\mathcal{U}(X)$.

Note that if $\theta_{e}$ is $1-1$, then it follows that $\theta$ is also 1-1. Notice also that if the action of $A$ on $X$ is faithful (i.e., if $a X=0$ implies that $a=0$ ), then $\theta_{e}$ is 1-1. This follows from Corollary 1.3. If the action of $A$ on $X$ is 'completely 1-faithful' (that is, the norm of $a \in M_{n}(A)$ is achieved as the supremum of the norms of the action of $a$ on $X$ in a sensible way), then $\theta_{e}$ and $\theta$ in Corollary 2.3 are complete isometries.

Proposition 2.4. For an operator bimodule $X$, with the notations above, we have $I\left(\mathcal{U}_{e}(X)\right)=I(\mathcal{S}(X))$. If $X$ is an operator $A$-B-bimodule over $C^{*}$ algebras $A$ and $B$, which is faithful as a left and as a right module, and if $\mathcal{U}(X)$ is the triangular operator algebra associated with a CES representation of $X$, then $I(\mathcal{U}(X))=I(\mathcal{S}(X))$.

Proof. Clearly $\mathcal{U}_{e}(X) \subset I(\mathcal{S}(X))$. Any minimal $\mathcal{U}_{e}(X)$-projection [17] is the identity on $\mathcal{S}(X)$, and is therefore the identity map. The rest is clear.

Definition 2.5. Let $Y$ be an operator $A$-B-bimodule. We shall call $Y$ an $A$-B-injective bimodule provided for every pair of operator $A$-B-bimodules $V$ and $W$, with $V$ a submodule of $W$, each completely contractive $A$ - $B$ bimodule map $T: V \rightarrow Y$ extends to a completely contractive $A$ - $B$-bimodule map from $W$ to $Y$.

We should remark that the above definition corresponds to what was called in [16] a tight $A$-B-injective bimodule. 
The following result extends Wittstock's theorem [39] that an injective $\mathrm{C}^{*}$-algebra is an injective operator module over a unital $\mathrm{C}^{*}$-subalgebra. In the following we consider unital $\mathrm{C}^{*}$-algebras, but it is not difficult to remove the 'unital' hypothesis.

Theorem 2.6. Let $A$ and $B$ be unital $C^{*}$-algebras.

(i) If $Y$ is an operator space which is also an operator $A$-B-bimodule, then $Y$ is injective as an operator space if and only if $Y$ is an $A$-B-injective bimodule.

(ii) If $Y$ is an operator $A$-B-bimodule, then the operator space injective envelope $I(Y)$, is the operator $A$-B-bimodule injective envelope of $Y$. That is, $I(Y)$ is an $A$-B-injective bimodule which is rigid and essential, as an operator $A$-B-bimodule containing $Y$. Rigidity here, for example, means: Any completely contractive A-B-bimodule map $I(Y) \rightarrow I(Y)$, which is the identity on $Y$, is the identity on $I(Y)$.

Proof. One direction of (i) is obvious. Namely, suppose that $Y$ is $A$ - $B$ injective. By CES, $Y$ may be realized as an $A$ - $B$-submodule of some $B(K, H)$, where $H$ is a Hilbert $A$-module and $K$ is a Hilbert $B$-module. Indeed $B(K, H)$ is an operator $A$ - $B$-bimodule. By the $A$ - $B$-injectivity of $Y$, there is a completely contractive projection from $B(K, H)$ onto $Y$. Since $B(K, H)$ is injective as an operator space, so is $Y$.

The other direction of (i) is harder. In a previous version of this paper we had a proof which used almost all the results established until now. Instead we shall only use a few results from part 1, and 2.2 above. We will also use the fact, which is a simple consequence of Wittstock's original result, or Suen's modification of this result [36], that if $H$ is a Hilbert $A$-module, then for any Hilbert space $K$, we have that $B(K, H)$ is $A$-injective. Indeed their result gives that $B(H)$ is $A$-injective if $A \subset B(H)$ isometrically. However in the contrary case, one may use the following kind of trick: Pick a Hilbert space $H^{\prime}$ in which $A$ is faithfully represented, then one obtains a faithful representation of $A$ on $H \oplus H^{\prime}$. Then one may apply the Wittstock or Suen result to conclude that $B\left(H \oplus H^{\prime}\right)$ is $A$-injective, from which it is easy to see by compression that $B(H)$ is $A$-injective. See [6] Theorem 4.1 for another proof of this simple consequence.

Suppose that $Y$ is injective. Represent the $\mathrm{C}^{*}$-algebra $I(\mathcal{S}(Y))$ faithfully and non-degenerately on a Hilbert space; then the two diagonal projections determine a splitting of the Hilbert space as $H \oplus K$, say. So $I_{11}$ is a unital *-subalgebra of $\mathrm{B}(\mathrm{H})$, and so on. Now by injectivity there is a completely positive projection $\phi$ from $B(H \oplus K)$ onto $I(\mathcal{S}(Y))$. As in the proof of 1.7, the Choi Lemma implies that this projection is an $I(\mathcal{S}(Y))$-module map, and that $\phi$ decomposes as a $2 \times 2$ matrix of maps. Let $\psi$ be the ' $1-2$ corner' of $\phi$. Thus $\psi: B(K, H) \rightarrow Y$ is a completely contractive projection onto $Y$, and its easy to see, as in 1.7 , that $\psi$ is a left $I_{11}$-module map. However 
if $Y$ is an operator $A$ - $B$-bimodule, then by 2.2 above there is a unital *homomorphism $\theta: A \rightarrow I_{11} \subset B(H)$ implementing the left module action. Hence $H$ is a Hilbert $A$-module, via $\theta$. Since $\theta$ maps into $I_{11}$, we see that the projection $\psi$ is a left $A$-module map onto $Y$. Similarly, $\psi$ is a right $B$-module map onto $Y$. Since $B(K, H)$ is $A$-B-injective, so is $Y$.

(ii) is obvious, given (i) and the fact, observed earlier, that the $A$ - $B$-action on $Y$ extends to make $I(Y)$ an operator $A$ - $B$-bimodule.

The proof in fact shows that any injective operator space $X$ is an $I_{11}-I_{22^{-}}$ injective operator bimodule.

At this point we may give another proof of the self-duality of $I(X)$ : By 2.6 (i), $I(X)$ is injective in the category of right $\mathrm{C}^{*}$-modules over $I_{22}$. Now appeal to [25] Prop. 3.10.

The following corollary of 1.8 generalizes a standard fact for Hilbert spaces:

Corollary 2.7. Let $Y$ be a right $C^{*}$-module over a $C^{*}$-algebra $A$, which (with respect to its canonical operator space structure) is an injective operator space. Then:

(i) $Y$ is a self-dual $C^{*}$-module over $A$.

(ii) $B_{A}(Y)=\mathbb{B}_{A}(Y)$.

(iii) Every bounded module map $Y \rightarrow Z$ is adjointable, for any other $C^{*}$ module $Z$.

(iv) $\mathbb{B}_{A}(Y)$ is an injective $C^{*}$-algebra.

Proof. We may suppose w.l.o.g. that $Y$ is a full $\mathrm{C}^{*}$-module over $A$. Thus we may regard $Y$ as a $\mathbb{K}(Y)-A$-imprimitivity bimodule. Let $Z=I(Y)=$ $Y$ equipped with its $\mathcal{C}(Y)-\mathcal{D}(Y)$-imprimitivity bimodule structure. Here $\mathcal{C}(Y) \subset I_{11}$ as usual. From [19] or A.7 in [7], we know that $Z \cong Y$, as imprimitivity bimodules. Thus $\mathbb{B}_{A}(Y) \cong I_{11}$, giving (iv). Similarly we get (i). It is well-known, and fairly obvious, that (i) implies (ii) and (iii).

The following result must be well-known. Since we cannot give a precise reference, we prove it:

Proposition 2.8. If $A$ is a $C^{*}$-algebra without identity, then the operator space injective envelope $I(A)$ is a unital $C^{*}$-algebra. Indeed $I(A)=I\left(A^{1}\right)$, where $A^{1}$ is the unitization of $A$.

Proof. Represent $A$ non-degenerately and faithfully on a Hilbert space $H$. If $\left\{e_{i}\right\}$ is a contractive approximate identity for $A$, then $e_{i} \rightarrow I_{H}$ in the SOT. We also have $I_{H} \in A^{1} \subset B(H)$. Let $\Phi: B(H) \rightarrow I(A) \subset B(H)$ be a minimal $A$-projection. So $\Phi$ is a completely contractive idempotent map whose range contains $A$. If $\Phi(I)=I$ we would be done, since in that case $\Phi$ is completely 
positive, and then we can deduce that $I(A)$ is a unital $\mathrm{C}^{*}$-algebra as in the introduction (i.e., by the Choi-Effros result quoted there).

In order to see that $\Phi(I)=I$, we choose $\zeta \in H$ with $\|\zeta\|=1$. Let $\phi(T)=\langle\Phi(T) \zeta, \zeta\rangle$, for $T \in B(H)$. Then $\|\phi\| \leq 1$ and $\phi\left(e_{i}\right) \rightarrow 1$. It is no doubt well-known and easy to see that this implies that $\phi(I)=1$. One way to do this is to write $\phi(T)=\langle\pi(T) \eta, \xi\rangle$, where $\pi$ is a unital *representation of $B(H)$ on a Hilbert space $K$, and where $\eta, \xi \in \operatorname{BALL}(K)$. Set $K^{\prime}=[\pi(A) K]$, and let $P$ be the projection onto $K^{\prime}$. Then it is easy to see that the net $\pi\left(e_{i}\right)$ has $P$ as a WOT limit point. For if $T$ is a WOT limit point of $\pi\left(e_{i}\right)$, then for $x, y \in K$ we have

$$
\langle T x, y\rangle=\lim _{i}\left\langle\pi\left(e_{i}\right) x, y\right\rangle=\lim _{i}\left\langle\pi\left(e_{i}\right) x, P y\right\rangle=\langle x, P y\rangle=\langle P x, y\rangle .
$$

Thus $\langle P \eta, \xi\rangle=\lim \phi\left(e_{i}\right)=1$. By the converse to Cauchy-Schwarz, $\xi=$ $P \eta=P^{2} \eta=P \xi$. Thus

$$
\phi(I)=\langle\eta, \xi\rangle=\langle\eta, P \xi\rangle=\langle P \eta, \xi\rangle=1 .
$$

In $[\mathbf{1 6}]$, it is shown that for a $\mathrm{C}^{*}$-algebra $A$, there is a canonical inclusion $L M(A) \subset I(A)$. Indeed, for any essential ideal $K$ of $A$, we have $L M(K) \subset$ $I(A)$. We can offer another proof of these results using our methods. Firstly, we just saw that if $X=A$ considered as an operator space, then $I(X)$ is a unital injective $\mathrm{C}^{*}$-algebra. From this it follows from abstract principles that $I(\mathcal{S}(X))=I(\mathcal{S}(I(X)))=M_{2}(I(A))$ (see for example [7] $4.18(\mathrm{i})$ ). Thus in this case $I_{11}=I(A)$. Hence

$$
L M(A)=M_{l}(A)=I M_{l}(A) \subset I(A) .
$$

If $K$ is an essential ideal in $A$, then as we just proved, $M(K) \subset I(K)$. Thus we have

$$
K \subset A \subset M(K) \subset I(K) .
$$

Any minimal $A$-projection on $I(K)$, is a $K$-projection, and is therefore equal to the identity map, by rigidity of $I(K)$. Thus $I(K)=I(A)$. By the first part, $L M(K) \subset I(K)=I(A)$. This gives the result we need.

Notice from the above, that since $A$ is an essential ideal in $M(A)$, we have $I(A)=I(M(A))=I\left(A^{1}\right)$.

The same is true even if $A$ is a nonselfadjoint operator algebra with contractive approximate identity, with some modifications in proof.

If $X$ is a $\mathrm{C}^{*}$-module we see from results above that

$$
I(\mathbb{B}(X))=I(M(\mathbb{K}(X)))=I(\mathbb{K}(X)),
$$

where $\mathbb{K}(X)$ is the so-called 'imprimitivity $\mathrm{C}^{*}$-algebra' of so-called 'compact' maps on $X$. Does this equal $I_{11}$ in this case? Certainly $\mathbb{K}(X) \subset I_{11}$ as a *-subalgebra, so that $I(\mathbb{K}(X)) \subset I_{11}$. 
Since this paper was written further advances have been made, for example in the papers $[\mathbf{8}, \mathbf{9}]$.

\section{References}

[1] E.M. Alfsen and E.G. Effros, Structure in real Banach spaces I and II, Ann. of Math., 96 (1972), 98-173, MR 50 \#5432, Zbl 248.46019.

[2] W.B. Arveson, Subalgebras of $C^{*}$-algebras, Acta Math., 123 (1969), 141-224, MR 40 \#6274, Zbl 194.15701.

[3] , Subalgebras of $C^{*}$-algebras II, 128 (1972), 271-308, MR 52 \#15035, Zbl 245.46098.

[4] E. Behrends, M-structure and the Banach-Stone theorem, Lecture Notes in Math., 736, Springer-Verlag, Berlin, (1979), MR 81b:46002, Zbl 436.46013.

[5] D.P. Blecher, Geometry of the tensor product of $C^{*}$-algebras, Math. Proc. Camb. Philos. Soc., 104 (1988), 119-127, MR 89g:46094, Zbl 668.46027.

[6] Modules over operator algebras and the maximal $C^{*}$-dilation, J. Funct. Analysis, 169 (1999), 251-288, MR 2000j:47122, Zbl 947.46041.

[7] _ The Shilov boundary of an operator space and the characterization theorems, to appear in J. Funct. Analysis.

[8] _ Multipliers and dual operator algebras, to appear J. Funct. Analysis.

[9] D. P. Blecher, E. G. Effros and V. Zarikian, One-sided M-ideals and multipliers in operator spaces. I, Preprint (December 2000).

[10] D.P. Blecher, Z.J. Ruan and A.M. Sinclair, A characterization of operator algebras, J. Funct. Analysis, 89 (1990), 188-201, MR 91b:47098, Zbl 714.46043.

[11] M.-D. Choi, A Schwarz inequality for positive linear maps on $C^{*}$-algebras, Illinois J. Math., 18 (1974), 565-574, MR 50 \#8089, Zbl 293.46043.

[12] M.-D. Choi and E.G. Effros, Injectivity and operator spaces, J. Funct. Analysis, 24 (1977), 156-209, MR 55 \#3814, Zbl 341.46049.

[13] E. Christensen, E.G. Effros and A.M. Sinclair, Completely bounded multilinear maps and $C^{*}$-algebraic cohomology, Inv. Math., 90 (1987), 279-296, MR 89k:46084, Zbl 646.46052.

[14] E. Christensen and A. Sinclair, Representations of completely bounded multilinear operators, J. Funct. Analysis, 72 (1987), 151-181, MR 89f:46113, Zbl 622.46040.

[15] E. G. Effros and Z. J. Ruan, Operator Spaces, Oxford University Press, Oxford, 2000.

[16] M. Frank and V.I. Paulsen, Injective envelopes of $C^{*}$-algebras as operator modules, preprint (April, 1999).

[17] M. Hamana, Injective envelopes of operator systems, Publ. R.I.M.S. Kyoto Univ., 15 (1979), 773-785, MR 81h:46071, Zbl 436.46046.

[18] , Symposium Lecture, R.I.M.S. Kyoto Univ. Surikaisekikenkyusho, Kokyuroku, 560 (May 1985), 128-141.

[19] _ Injective envelopes of dynamical systems, preprint, April 1991. Some of this appeared under the same title, in 'Operator algebras and operator theory,' Pitman Research Notes, 271 (1992), 69-77, Longman, MR 94a:46100a, Zbl 788.46066. 
[20] - Triple envelopes and Silov boundaries of operator spaces, Math. J. Toyama University, 22 (1999), 77-93.

[21] H.E. Lacey, The isometric theory of classical Banach spaces, Grundlehren Band, 208, Springer-Verlag, 1974, MR 58 \#12308, Zbl 285.46024.

[22] E.C. Lance, Hilbert $C^{*}$-modules - A toolkit for operator algebraists, London Math. Soc. Lecture Notes, Cambridge University Press, 1995, MR 96k:46100, Zbl 822.46080.

[23] C. Le Merdy, Representation of a quotient of a subalgebra of $B(X)$, Math. Proc. Camb. Philos. Soc., 119 (1996), 83-90, MR 97e:47074, Zbl 847.46029.

[24] H. Lin, Bounded module maps and pure completely positive maps, J. Operator Theory, 26 (1991), 121-138, MR 94f:46071, Zbl 791.46032.

[25] _ Injective Hilbert $C^{*}$-modules, Pacific J. Math., 154 (1992), 131-164, MR 93c:46110, Zbl 799.46057.

[26] B. Magajna, Hilbert modules and tensor products of operator spaces, Linear Operators, Banach Center Publ., 38, Inst. of Math. Polish Acad. Sci., (1997), 227-246, MR 98h:46060, Zbl 879.46024.

[27] W. Paschke, Inner product modules over $B^{*}$-algebras, Trans. Amer. Math. Soc., 182 (1973), 443-468, MR 50 \#8087, Zbl 265.46056.

[28] V.I. Paulsen, Every completely polynomially bounded operator is similar to a contraction, J. Functional Analysis, 55 (1984), 1-17, MR 86c:47021, Zbl 557.46035.

[29] _ Completely bounded maps and dilations, Pitman Research Notes in Math., Longman, London, 1986, MR 88h:46111, Zbl 614.47006.

[30] V.I. Paulsen and R.R. Smith, Multilinear maps and tensor norms on operator systems, J. Functional Analysis, 73 (1987), 258-276, MR 89m:46099, Zbl 644.46037.

[31] G. Pedersen, $C^{*}$-algebras and their automorphism groups, Academic Press, 1979, MR 81e:46037, Zbl 416.46043.

[32] G. Pisier, An introduction to the theory of operator spaces, preprint.

[33] Y-t. Poon and Z.J. Ruan, Operator algebras with contractive approximate identities, Canadian J. Math., 46 (1994), 397-414, MR 95d:47057, Zbl 816.47048.

[34] M.A. Rieffel, Morita equivalence for operator algebras, Proceedings of Symposia in Pure Mathematics, Part 1, 38 (1982), 285-298, MR 84k:46046, Zbl 541.46044.

[35] Z.J. Ruan, Injectivity of operator spaces, Trans. A.M.S., 315 (1989), 89-104, MR 91d:46078, Zbl 669.46029.

[36] C-Y. Suen, Completely bounded maps on $C^{*}$-algebras, Proc. A.M.S., 93 (1985), 81-87, MR 86d:46053, Zbl 553.46040.

[37] M. Tomita, Spectral theory of operator algebras II, Mathematical Journal of Okayama University, 10 (1962), 19-60.

[38] N.E. Wegge-Olsen, K-theory and $C^{*}$-algebras, Oxford Univ. Press, 1993, MR 95c:46116, Zbl 780.46038.

[39] G. Wittstock, Extension of completely bounded $C^{*}$-module homomorphisms, in 'Proceedings, Conf. on Operator Algebras and Group Representations", Neptum 1980, 238-250, Pitman, New York, 1984, MR 85i:46080, Zbl 535.46003.

[40] M. Youngson, Completely contractive projections on $C^{*}$-algebras, Quart. J. Math. Oxford, 34 (1983), 507-511, MR 85f:46112, Zbl 542.46029.

[41] C. Zhang, Representations of operator spaces, J. Oper. Th., 33 (1995), 327-351, MR 96h:46092, Zbl 837.47037. 
Received October 5, 1999 and revised January 31, 2000. Portions of this work were presented at the Canadian Operator Theory and Operator Algebras Symposium, May 20, 1999. The authors were supported by a grant from the NSF.

Department of Mathematics

UNIVERSITY OF HOUSTON

Houston, TX 77204-3476

E-mail address: dblecher@math.uh.edu

Department of Mathematics

UNIVERSITY OF HOUSTON

Houston, TX 77204-3476

E-mail address: vern@math.uh.edu 